\title{
Management of Cellular Digital Packetized Data (CDPD) Networks
}

\author{
Moderator: Jock EMBRY, Opening Technologies, U.S.A.
}

The Cellular Digital Packet Data (CDPD) Network extends existing data networks to mobile data devices, by using radio channels and cell sites already in place for Advanced Mobile Phone Service (AMPS). Currently being deployed throughout North America and other regions, CDPD services will enable a wide variety of applications for wireless users, such as e-mail, dispatching, mobile query, portable point-of-sale terminals, etc. The CDPD Specification calls for both existing technology, such as off the self routers, and new network elements unique to CDPD. The management part of the CDPD Specification is based on OMNIPoint 1, and adds additional ensembles and managed objects specific to CDPD.

This panel will discuss the issues and challenges associated with managing the CDPD Network, such as agent deployment, integration with existing management systems, tradeoff between proprietary and standards based solutions, and interoperability between service providers. 